\title{
Article
}

\section{Care of the critically ill patient with a tracheostomy}

Billington, John James and Luckett, Alison

Available at http://clok.uclan.ac.uk/29795/

Billington, John James and Luckett, Alison (2019) Care of the critically ill patient with a tracheostomy. Nursing Standard, 34 (9). pp. 59-65. ISSN 00296570

It is advisable to refer to the publisher's version if you intend to cite from the work. http://dx.doi.org/10.7748/ns.2019.e11297

For more information about UCLan's research in this area go to

http://www.uclan.ac.uk/researchgroups/ and search for < name of research Group>.

For information about Research generally at UCLan please go to http://www.uclan.ac.uk/research/

All outputs in CLoK are protected by Intellectual Property Rights law, including Copyright law. Copyright, IPR and Moral Rights for the works on this site are retained by the individual authors and/or other copyright owners. Terms and conditions for use of this material are defined in the policies page.

\section{CLoK}

Central Lancashire online Knowledge www.clok.uclan.ac.uk 


\title{
Nursing Standard
}

\section{cardiorespiratory / CPD}

\section{Why you should read this article:}

- To improve your knowledge of the various equipment and procedures required when caring for a patient with a tracheostomy

- To understand the role of the nurse in tracheostomy insertion and maintenance

- To count towards revalidation as part of your 35 hours of CPD, or you may wish to write a reflective account (UK readers)

- To contribute towards your professional development and local registration renewal requirements (non-UK readers)

\section{Care of the critically ill patient with a tracheostomy}

John Billington and Alison Luckett

\author{
Citation \\ Billington J, Luckett A (2018) Care of the critically ill patient with a tracheostomy. Nursing Standard. doi: \\ 10.7748/ns.2018.e11297
}

Peer review

This article has been subject to external double-blind peer review and checked for plagiarism using automated software

Correspondence

jjbillington@uclan.ac.uk

@Johnjbilly2

Conflict of interest

None declared

Accepted

29 August 2018

Published online

$x x x x x$

\begin{abstract}
Tracheostomy management is increasingly necessary in critical care units and general wards. Therefore, it is important that nurses are equipped with the appropriate knowledge and skills to meet the individual needs of patients with a tracheostomy safely and competently. This article aims to enhance nurses' understanding of the potential challenges that patients with a tracheostomy may experience, and to guide nurses in providing effective care and support to these patients. It outlines the care that should be provided for patients with a tracheostomy who are critically ill, including methods of humidification and endotracheal suctioning. This article also discusses the effects that a tracheostomy may have on a patient's communication and psychological well-being, and explains the actions that nurses should take in an emergency and if complications occur.
\end{abstract}


John Billington, senior lecturer, School of Health Sciences, University of Central Lancashire, Preston, England; Alison Luckett, senior lecturer, School of Nursing, University of Central Lancashire, Preston, England

\section{Keywords}

critical care, emergency care, intensive care, respiratory, temporary tracheostomy, tracheostomy, tracheostomy tube

Key points

- Nurses must understand the immediate post-insertion and long-term management required in patients with a tracheostomy if they are to provide safe and effective care

- Critical care units in the UK use tracheostomy tubes from a variety of manufacturers, so it is important that nurses are familiar with the type and size of tube inserted (Intensive Care Society 2014).

- The nurse's role in tracheostomy insertion is usually to gather the necessary equipment and to ensure the correct checklists are completed (Plowright 2014)

- The psychological well-being of patients with a tracheostomy should be considered as important as the physical interventions, because patients without a speaking valve will be unable to speak, and this can cause them anxiety and frustration

\section{Aims and intended learning outcomes}

This article aims to explore the evidence related to the nursing care required for a patient with a tracheostomy. It also considers the types of tracheostomy tubes used in patients who are critically ill and discusses the essential nursing care and psychological support that they may require. The article also describes the considerations necessary when discharging a patient with a tracheostomy from a critical care unit to a general ward. After reading this article and completing the time out activities you should be able to:

" Identify the reasons why patients might require a tracheostomy and the types of tracheostomy tubes available.

» Understand the role of the nurse in the insertion and management of a percutaneous tracheostomy.

» Discuss the care and management interventions that can support patient safety in those with a tracheostomy, such as humidification and endotracheal suctioning.

» Explain the emergency situations and complications that may occur in patients with a tracheostomy, and how these can be managed effectively.

\section{Introduction}

The number of patients who are critically ill who have a tracheostomy is unknown, although it is widely noted in the literature that the procedure is common, and its prevalence is increasing (Intensive Care Society (ICS) 2014). Tracheostomy is rarely the primary procedure being undertaken for a patient, and is usually undertaken in response to clinical deterioration. Nurses must understand the immediate post-insertion and long-term management required in patients with a tracheostomy if they are to provide safe and effective care. However, there are concerns that the standard of tracheostomy care provided is often inadequate. The National Confidential Enquiry into Patient Outcome and Death (NCEPOD) (2014) found that there was potential for improvement in tracheostomy care related to cuff management, the monitoring and frequency of clinical observations, tube selection, and the weaning process.

The trachea links the larynx to the lungs and in an adult is approximately $1.8 \mathrm{~cm}$ in diameter and $10 \mathrm{~cm}-12 \mathrm{~cm}$ in length. The trachea is comprised of C-shaped rings of cartilage that stiffen and hold the trachea open. Ligaments and bundles of smooth muscle hold the cartilage rings together. The trachea is lined with a ciliated pseudostratified columnar epithelium with goblet cells, which progressively changes as the airways branch [Q1 Can you explain what you mean by 'progressively changes' here - are you referring to the make-up of cells in the trachea, which changes as the airways branch, and if so, how do they change?] (Pocock et al 2013).

A tracheostomy is a surgical procedure that is undertaken to create an opening (stoma) in the anterior wall of the trachea. To maintain the patency of the stoma and thus the airway, a percutaneous tracheostomy tube is inserted through the opening 
and into the trachea (Figure 1) (Dawson 2014). A tracheostomy may be undertaken as a temporary or permanent measure. A temporary tracheostomy is a life-saving intervention that is undertaken when there is a structural or functional obstruction of airflow, and is usually for short-term use. A permanent tracheostomy is non-weanable and cannot be removed. It is inserted as a means of providing long-term ventilation to patients who have progressive or permanent conditions such as cancer of the larynx, motor neurone disease and severe head injury (Everitt 2016).

Percutaneous dilatational tracheostomy (PDT) is the most commonly undertaken procedure for tracheostomy insertion in critical care units, and it has been estimated that up to 15,000 PDT insertions take place annually in England (National Tracheostomy Safety Project (NTSP) 2013, ICS 2014).

\section{Figure 1. Anatomical placement of percutaneous tracheostomy}

[Q2 please could you check that the redrawn figure below is medically accurate and confirm if you are happy with it, or indicate if there are any changes that need to be made]

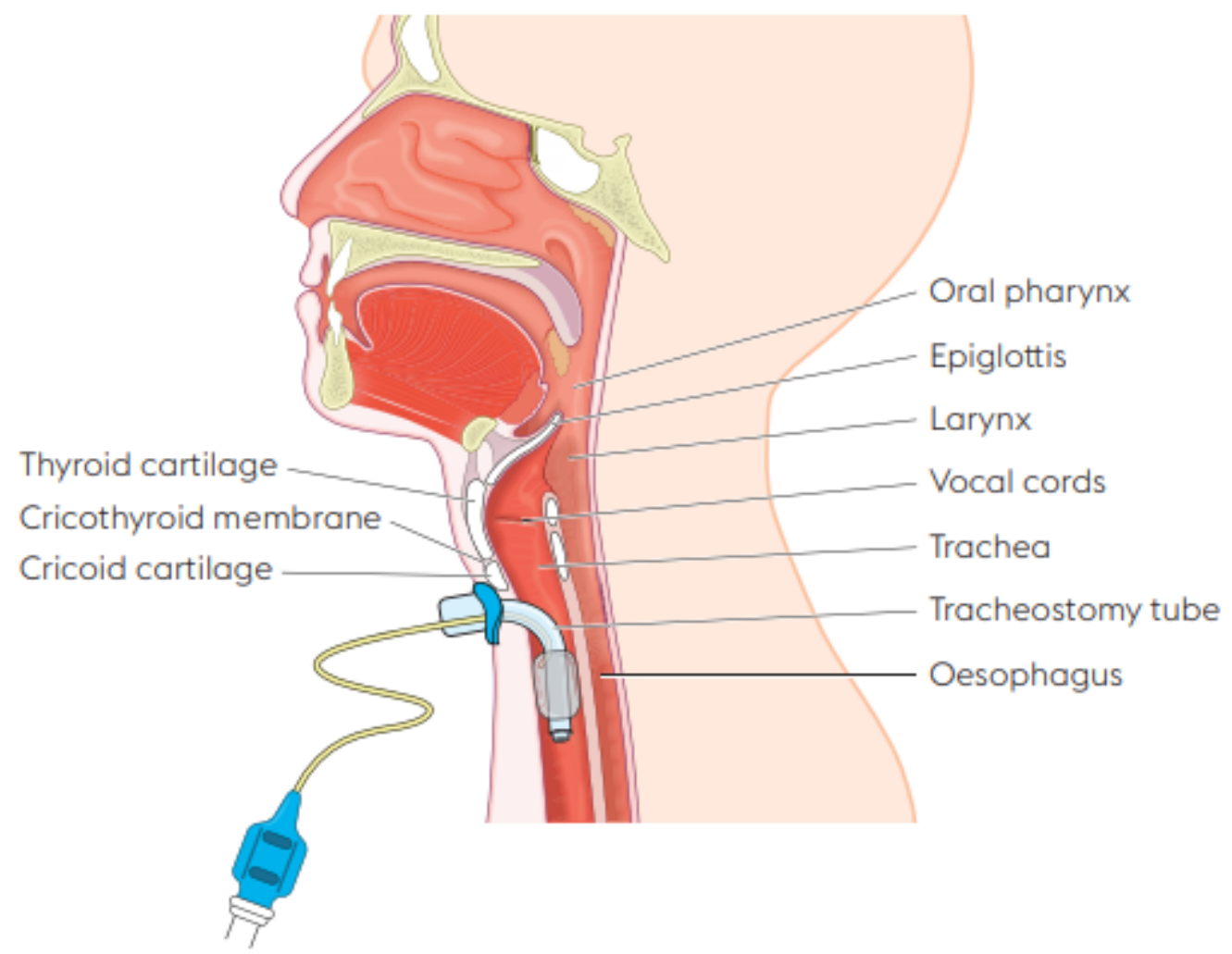

\section{TIME OUT 1}

Consider the various types of tracheostomy tubes that are used in your area of practice and note the indications for their usage. Refer to the manufacturer's guidelines and note what they recommend regarding the sizing, storage and maintenance of tracheostomy tubes

\section{Types of tracheostomy tube}

Critical care units in the UK use tracheostomy tubes from a variety of manufacturers, so it is important that nurses are familiar with the type and size of tube inserted (ICS 2014). Information can be found on the leaflet accompanying the tracheostomy tube, the manufacturer's website or in Chapter 9 of the Royal Marsden Manual of Clinical Nursing Procedures (Dougherty and Lister 2015). Tracheostomy tubes are either cuffed or uncuffed, and fenestrated or unfenestrated. A cuffed tracheostomy tube has an inflatable cuff that creates an airtight seal around the tube and the trachea, thereby maintaining the air delivered from a mechanical ventilator to a patient's lungs. Deflating the cuff makes it possible for the patient to create airflow in the upper airways and therefore cough. Deflating the cuff is also a technique commonly used to encourage 
weaning from mechanical ventilation. Fenestrated tracheostomy tubes have openings that allow air to pass freely and enable patients to communicate with the use of a speaking valve (ICS 2014).

Single and double cannula tracheostomy tubes are also available. Double cannula tubes are preferred in patients who are mechanically ventilated, as a safety measure against occlusion by secretions (ICS 2014). Secretions can adhere to the internal lumen of a tracheostomy tube and significantly reduce the inner lumen diameter, increasing the effort involved in breathing and/or obstructing the patient's airway. The removable inner cannula can be changed and cleaned frequently and quickly, thereby reducing the risk of occlusion.

The size of the tracheostomy tube can be found printed on the flange of the device. Tube size is determined by the patient's anatomy, and should be approximately three quarters of the patient's tracheal diameter; sizes 6 or 8 are most commonly used in adult patients (Schreiber 2015). Adjustable flange tubes should be considered for use in patients who are obese or those with anatomical distortion (ICS 2014, Plowright 2014).

Tracheostomy tubes with subglottic suction ports have been introduced because they have the potential to reduce the risk of ventilator-associated pneumonia (VAP) from the migration of secretions located above the cuff into the lower respiratory tract (NTSP 2013). Undertaking subglottic suction at a minimum of two hourly intervals is one of six high-impact intervention actions required within the Department of Health (DH) (2010) care bundle to reduce VAP.

Ledgerwood et al (2013) undertook a small-scale comparison study of 18 patients with tracheostomy, half of whom underwent continuous subglottic suction, while the other half did not. They found that there was a $45 \%$ reduction in the incidence of VAP in the suction group. This resulted in a mean reduction in length of hospital stay of eight days. Mao et al's (2016) meta-analysis of 20 randomised controlled trials found that both intermittent and continuous subglottic suction decreased the incidence of VAP. Mao et al's (2016) study was undertaken on a significantly larger scale $(N=3,544)$ than Ledgerwood et al's (2013) study, and conversely found that subglottic suction did not reduce length of hospital stay and mortality.

\section{TIME OUT 2}

List the equipment you would need to gather in preparation for the insertion of a percutaneous tracheostomy. What nursing care would need to be provided when preparing the patient for the procedure?

\section{Role of the nurse in tracheostomy insertion}

The nurse's role in tracheostomy insertion is usually to gather the necessary equipment and ensure the correct checklists are completed (Plowright 2014). PDT should be classed as a surgical procedure that requires informed consent from the patient, where possible, as well as completion of pre-operative checklists (ICS 2014, NCEPOD 2014). The nurse is required to prepare for PDT insertion by completing tasks such as timely cessation of nasogastric feeding and ensuring blood-clotting values are obtained before the procedure (Acharya and Nanjangud 2014). Patients receiving high concentrations of supplemental oxygen or high inspiratory pressures may not tolerate the hypoventilation and derecruitment (collapse of aveoli) experienced during insertion (NTSP 2013). For this reason, tracheostomy is not recommended for patients who are mechanically ventilated who require fraction of inspired oxygen $\left(\mathrm{FiO}_{2}\right)>0.6$ and positive end-expiratory pressure (PEEP) $>10 \mathrm{cmH}_{2} \mathrm{O}$ (ICS 2014). During the procedure, the nurse must undertake close observation of oxygen saturations, continuous cardiac monitoring via electrocardiogram (ECG), invasive blood pressure and capnography monitoring (monitoring of the concentration of carbon dioxide in the respiratory gases) (ICS 2014).

Following the insertion of a tracheostomy tube, it is vital that the nurse ensures an appropriate bedhead sign is clearly visible and correctly completed. The bedhead sign should include the patient's name, hospital number, date of insertion of tracheostomy, type of tracheostomy, tube size and emergency contact details (NTSP 2013). The nurse should be aware that the stoma tract in PDT insertions is usually established within 7-10 days of insertion; therefore, if the tracheostomy tube becomes displaced before this timeframe, rapid reinsertion will be challenging (ICS 2014). 


\section{Tracheostomy care and management interventions}

\section{Humidification}

A tracheostomy bypasses the body's normal upper airway mechanisms for humidification, filtration and warming of inspired gases. This results in increased viscosity of mucus secretions, which depresses ciliary function and, in turn, mucociliary transport (Russell 2005). This can lead to an increased risk of infection, impaired secretion removal and microatelectasis (alveolar collapse in the presence of patent airways) (NTSP 2013). Failure to provide adequate humidification to address these issues can lead to obstruction of the major airways and tracheostomy tube blockage. There is also an increased risk of atelectasis (lung collapse resulting in reduced gas exchange), consolidation (lung tissue that is filled with liquid rather than air), pneumonia and blocking of the tracheostomy tube. To prevent this, humidification should be provided at all times and nurses should take into consideration the retention of tenacious secretions. [Q3 Can you explain what you mean here, by 'take into consideration', i.e. do you mean that nurses should attempt to remove tenacious secretions; how would they do this?]

There are various methods for the provision of humidification, which depend on the patient's individual needs; however, the most important factor is to ensure the patient receives adequate systemic hydration to ensure they do not become dehydrated and risk developing thick, dry secretions (NTSP 2013). Humidification can be provided by using heated water humidifiers in which gases pass across or over a heated water bath (Selvaraj 2010) [Q4 please add to references list], or by using heat- and moisture-exchange filters, where heated and humidified gas is inhaled by the patient.

\section{TIME OUT 3}

Think about an occasion where you have undertaken suctioning on a patient. Reflect on the areas of practice such as pre-oxygenation, recovery from desaturation and pain management. Can you identify any ways in which these areas could be improved?

\section{Endotracheal suctioning}

Endotracheal suctioning is a component of bronchial hygiene therapy and mechanical ventilation. It involves the mechanical aspiration of pulmonary secretions from a patient with an artificial airway in place. The procedure involves patient preparation, the suctioning itself and follow-up care. Since patients with a temporary tracheostomy will have an impaired ability to clear their own secretions, nurses must have the knowledge and skills to be able to undertake suctioning safely and confidently. Some patients will receive nebulised solutions such as $0.9 \%$ sodium chloride as a means of loosening secretions and so that the patient can clear any mucous plugs. The patient's sputum characteristics [Q5 could you explain briefly what you mean by 'sputum characteristics', i.e. do you mean whether the sputum is too thick or too thin?] should be assessed at each suction procedure. The aim of suctioning is to clear the airway and aid normal breathing without causing any trauma or hypoxia (ICS 2014).

Suctioning involves the placement of a suction catheter through the artificial airway into the trachea and the application of negative pressure as the catheter is being withdrawn. A sterile technique must be used. Suction pressure should be set as low as possible, yet be able to effectively clear secretions. Selection of the appropriate size of suction catheter is important to reduce the risk of trauma and hypoxia. As a guide, the diameter of the suction catheter should not exceed one-half of the internal diameter of the tracheostomy tube. The following formula can be used to determine the appropriate size of suction catheter (Dougherty and Lister 2015): 2 x (size of tracheostomy tube - 2) = suction catheter size (Fg). For example, for a tube with an internal diameter of $8 \mathrm{~mm}$, this would be $2 \times(8-2)=12 \mathrm{Fg}$.

The suction catheter should be inserted to the point where the patient coughs, or to $0.5 \mathrm{~cm}-1.0 \mathrm{~cm}$ below the level of the tracheostomy tube, then it should be withdrawn half a centimetre before suction is applied; this prevents trauma to the carina (the ridge at the base of the trachea) (ICS 2014). Overall, any suctioning event should last no longer than 10-15 seconds (White et al 2010). The suctioning event can be painful for patients; therefore, it is important for the nurse to inform the patient of the need for the procedure and to administer adequate pain relief before the procedure is undertaken, as well as allaying any distress or anxiety. It is also important for the nurse to monitor the patient's condition after suctioning, by 
observing their respiratory rate and pattern, oxygen saturations, heart rate and work of breathing closely for 15 minutes (Dougherty and Lister 2015).

\section{TIME OUT 4}

Locate your healthcare organisation policy or guideline on the care of patients with a tracheostomy. What is recommended regarding the cleaning of the inner tube and cuff management?

\section{Inner tube cleaning and disposable inner cannulas}

Disposable, single-use inner tubes are now widely available, which eradicate the need for regular inner tube cleaning and have the potential to reduce cross infection (Dougherty and Lister 2015). Nurses must ensure that replacement inner tubes in the appropriate size are available at the patient's bedside at all times. Regular inner cannula checks should take place every four hours at a minimum to ensure tube patency (NCEPOD 2014). Patients who produce copious amounts of secretions are at high risk of tube occlusion and may require the inner cannula to be changed more frequently.

NTSP (2013) guidelines recommend inner tube cleaning of non-disposable inner cannulas at least once per eight-hour shift, with the NCEPOD (2014) report finding that the most common frequency of cleaning in critical care units is every four hours. If an inner cannula requires cleaning, a clean or aseptic technique should be used depending on the local critical care unit policy. Nurses must be aware that the procedure will result in a short period of time when oxygen supply and pressure support in patients who are mechanically ventilated will be interrupted; therefore, close observation of the patient's respiratory status during and immediately after the procedure is vital (Dougherty and Lister 2015).

\section{Cuff management}

Cuffed inner tubes have a high-volume, low-pressure design to aid even distribution of pressure against the tracheal wall (Dougherty and Lister 2015). Cuff pressure should not exceed $25 \mathrm{cmH}_{2} \mathrm{O}$, since excessive cuff pressure could indicate that the tube is too small or malpositioned, which could lead to tracheal stenosis (narrowing) (ICS 2014, Dougherty and Lister 2015). The NTSP (2013) guidelines recommend that cuff pressure should be measured at least once per shift; however, the DH (2010) care bundle aimed at reducing VAP, and local critical care unit guidelines, often recommend cuff checks every 24 hours. NCEPOD (2014) found that cuff pressure measurements immediately following tracheostomy insertion were undertaken in $73 \%(n=1011 / 1379)$ of patients. Nurses should ensure that a manometer is present at the patient's bedside to measure cuff pressure, and report any concerns about cuff pressure to a suitably trained doctor.

When using a speaking valve with a cuffed tube, the cuff must always be deflated. Failure to do so will prevent the patient from exhaling and lead to asphyxiation and possible respiratory or cardiac arrest (ICS 2014). Additional care should be taken when using a speaking valve with an uncuffed, unfenestrated tube because there may not be enough space between the trachea wall and tube for sufficient air to pass freely (Dougherty and Lister 2015).

\section{Importance of communication}

Patients who require a tracheostomy will be acutely unwell, and having a tracheostomy in situ increases their level of acuity. Communication difficulties may persist for patients the longer they require a tracheostomy; therefore, it is important that the nurse is able to interact with and reassure the patient effectively. If the patient has an uncuffed or a fenestrated tube with the cuff deflated, then verbal communication may be possible using a speaking valve.

The psychological well-being of patients with a tracheostomy should be considered to be as important as the physical interventions, because patients without a speaking valve will be unable to speak, and this can cause them anxiety and frustration. The purpose of effective communication is to provide the patient with a sense of identity and provide them with the opportunity to express themselves and share their feelings and needs.

There are several communication devices that can assist the patient in communicating effectively. The patient may be offered picture boards or letter boards that contain common phrases and messages to aid communication (Grossbach et al 
2011). The patient's family can also assist in expressing the patient's needs, which can enhance the therapeutic relationship and reduce overall frustration.

\section{Emergencies and complications}

The NCEPOD (2014) reported that $24 \%(n=461 / 1,956)$ of patients experienced a complication following the insertion of a tracheostomy while they were a patient in a critical care unit (NCEPOD 2014). Therefore, it has been recommended that all staff caring for patients who have a tracheostomy in situ receive training in how to respond appropriately to emergency situations, in line with NTSP (2013) or ICS (2014) guidelines.

Complications from tracheostomy can be divided into three categories: immediate, delayed and late (Box 1) (ICS 2014).

\section{Box 1. Complications from tracheostomy}

[Q6 times added from the ICS reference - is this ok?]

Immediate complications (peri-procedural period)

» Haemorrhage

» Primary displacement

» Pneumothorax

) Tube occlusion

» Surgical emphysema

" Loss of upper airway

\section{Delayed complications (post-operative period $<7$ days)}

» Tube occlusion

» Partial or complete displacement

" Infection of stoma site

» Pneumonia

ע) Ulceration of trachea

» Trachea-oesophageal fistula

» Haemorrhage (tissue erosion)

\section{Late complications (late post-operative period $>7$ days)}

» Excessive granulation of the trachea causing breathing difficulty on removal

» Tracheal dilation or stenosis

) Tube occlusion

» Haemorrhage

» Scar formation

(Adapted from Intensive Care Society 2014)

The ICS (2014) guidelines also outline 'red flags' that act as a warning sign of imminent issues, including:

»Inability to pass a suction catheter into the trachea.

» Altered capnography trace.

» Talking or noises heard over a cuffed tracheostomy tube.

» Frequent requirement for excessive cuff inflation to prevent air leaks.

» Pain or bleeding from the tracheostomy site.

»Visibly displaced tracheostomy tube.

Respiratory distress, increasing ventilator support anxiety, agitation and restlessness could also suggest the airway is compromised (ICS 2014).

NCEPOD (2014) identified the most serious complications of tracheostomy in critical care units and general wards, including haemorrhage, pneumothorax, accidental tube dislodgement or removal, and obstruction. NTSP (2013) have published robust guidelines for patients with a tracheostomy that should be available in all critical care units and general 
wards where patients might be transferred to, including the green emergency response algorithm, which is available at: www.tracheostomy.org.uk/storage/files/Patent\%20Airway\%20Algorithm.pdf

Nurses caring for patients with a tracheostomy in critical care units should be familiar with the algorithm and know where to locate it in an emergency.

\section{TIME OUT 5}

Consider the role of the critical care nurse when preparing to transfer the patient with a tracheostomy to the ward. What safety checks should be considered and how can these be implemented and documented?

\section{Decannulation and patient discharge from critical care}

The removal of a tracheostomy tube is known as decannulation. This is a two-person procedure and can be a nursing role in critical care, as long as the nurse undertaking the procedure is adequately trained to do so. Removing a patient's tracheostomy before they are transferred to a general ward environment is often preferred for safety reasons, so that the patient can be closely observed immediately post-decannulation, which may not be possible on a general ward. However, all patients who have had or still have a tracheostomy in situ on transfer require input from the multidisciplinary team (NCEPOD 2014). The multidisciplinary team should include ward nurses, physiotherapists, speech and language therapists, specialist nurses, the respiratory physicians, head and neck surgeons, and anaesthetists (NTSP 2013).

In preparation for decannulation, the cuff should be deflated, and a plug supplied with the tracheostomy tube can be applied to encourage patients to breathe around the tube before its removal (Dougherty and Lister 2015). Before removal of the tracheostomy, it is vital that the patient is alert, can maintain their own airway, breathe spontaneously, has a strong cough with the ability to expectorate, and is haemodynamically stable (NTSP 2013).

Discharging patients with a tracheostomy in situ from a critical care unit must be carefully considered. The receiving ward must have staff adequately trained to manage patients who have a tracheostomy in situ. Ideally, the tracheostomy tube should be uncuffed and have a double lumen to reduce the risk of occlusion (ICS 2014). However, the NCEPOD (2014) report identified that $95 \%(n=551 / 580)$ of patients discharged from a critical care unit still had a cuffed tracheostomy tube in situ. Once these patients had been discharged to a ward, cuff monitoring was less frequent and $28 \%(n=130 / 464)$ had the cuff continuously inflated, which could increase the risk of complications.

NTSP (2013) guidelines recommend that a portable box containing essential tracheostomy equipment (Box 2) is transferred with the patient from the critical care unit to the general ward. This equipment should remain at the patient's bedside for 48 hours following decannulation, in case of respiratory decline and the need for rapid reinsertion of a tracheostomy (NTSP 2013).

\section{Box 2. Essential tracheostomy equipment}

») Tracheal dilators

» Spare tracheostomy tubes - one that is the same type and size as the tube the patient has in situ, and one that is a size smaller than the tube the patient has in situ

» Tracheostomy disconnection wedge

»Oxygen administration device

»Yankauer sucker (oral suctioning tool)

» Stitch cutter

» $10 \mathrm{~mL}$ syringe

»Water soluble gel

»Cuff pressure gauge

» Tracheostomy tube holder

\section{Conclusion}

It is important that nurses understand the various reasons why patients who are critically ill might require a tracheostomy, and can provide evidence-based care that is safe and appropriate for the patient. Nurses should have the knowledge and skills 
necessary to provide optimum care to the patient with a tracheostomy, including humidification, endotracheal suctioning, inner tube cleaning and cuff management. Nurses should also be aware of their role in identifying and managing emergencies and complications when they occur, to preserve patient safety.

\section{TIME OUT 6}

Consider how caring for critically ill patients with a tracheostomy relates to The Code: Professional Standards of Practice and Behaviour for Nurses and Midwives (Nursing and Midwifery Council 2015) or, for non-UK readers, the requirements of your regulatory body

\section{TIME OUT 7}

Now that you have completed the article, reflect on your practice in this area and consider writing a reflective account: rcni.com/reflective-account

\section{References}

Acharya D, Nanjangud P (2014) Percutaneous dilatational tracheostomy. Indian Journal of Respiratory Care. 3, 2, 488-496.

Dawson D (2014) Essential principles: tracheostomy care in the adult patient. Nursing in Critical Care. 19, 2, 63-72. doi: 10.1111/nicc.12076.

Department of Health (2010) High Impact Intervention: Care Bundle to Reduce Ventilation-Association Pneumonia. http://webarchive.nationalarchives.gov.uk/20120118164404/http://hcai.dh.gov.uk/files/2011/03/2011-03-14-HII-Ventilator-AssociatedPneumonia-FINAL.pdf (Last accessed: 30 August 2018.)

Dougherty L, Lister S (Eds) (2015) The Royal Marsden Manual of Clinical Nursing Procedures. Ninth Edition. Wiley-Blackwell, Oxford.

Everitt E (2016) Tracheostomy 3: care of patients with permanent tracheostomy. Nursing Times. 112, 21-23, 20-22.

Grossbach I, Stranburg S, Chlan L (2011) Promoting effective communication for patients receiving mechanical ventilation. Critical Care Nurse. 31, 3, 46-60. doi: 10.4037/ccn2010728.

Intensive Care Society (2014) Standards for the Care of Adult Patients with a Temporary Tracheostomy. www.theawsomecourse.co.uk/ICS/ICS\%20Tracheostomy\%20standards\%20(2014).pdf (Last accessed: 30 August 2018.)

Ledgerwood LG, Salgado MD, Black H et al (2013) Tracheotomy tubes with suction above the cuff reduce the rate of ventilator-associated pneumonia in intensive care unit patients. Annals of Otology, Rhinology and Laryngology. 122, 1, 3-8. doi: $10.1177 / 000348941312200102$.

Mao Z, Gao L, Wang G et al (2016) Subglottic secretion suction for preventing ventilator-associated pneumonia: an updated metaanalysis and trial sequential analysis. Critical Care. 20, 1, 353. doi: 10.1186/s13054-016-1527-7.

National Confidential Enquiry into Patient Outcome and Death (2014) On the Right Trach? A Review of the Care Received by Patients who Underwent a Tracheostomy. www.ncepod.org.uk/2014report1/downloads/OnTheRightTrach_FullReport.pdf (Last accessed: 30 August 2018.)

National Tracheostomy Safety Project (2013) NTSP Manual 2013. NTSP, Manchester.

Nursing and Midwifery Council (2015) The Code: Professional Standards of Practice and Behaviour for Nurses and Midwives. NMC, London.

Plowright C (2014) Safe care of patients with tracheostomies. Nursing Times. 110, 31, 12-14.

Pocock G, Richards CD, Richards DA (2013) Human Physiology. Fourth Edition. Oxford University Press, Oxford.

Russell C (2005) Providing the nurse with a guide to tracheostomy care and management. British Journal of Nursing. 14, 8, 428-433. 
Schreiber ML (2015) Tracheostomy: site care, suctioning, and readiness. Medsurg Nursing. 24, 2, 121-124.

White AC, Kher S, O'Connor HH (2010) When to change a tracheostomy tube. Respiratory Care. 55, 8, 1069-1075. 